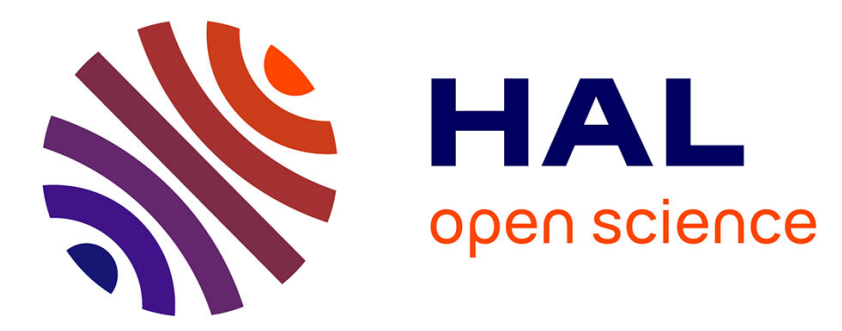

\title{
Visco-capillary response of gas bubbles probed by thermal noise atomic force measurement
}

Yuliang Wang, Binglin Zeng, Hadush Tedros Alem, Zaicheng Zhang, Elisabeth Charlaix, Abdelhamid Maali

\section{> To cite this version:}

Yuliang Wang, Binglin Zeng, Hadush Tedros Alem, Zaicheng Zhang, Elisabeth Charlaix, et al.. Viscocapillary response of gas bubbles probed by thermal noise atomic force measurement. Langmuir, 2018, 34 (4), pp.1371 - 1375. 10.1021/acs.langmuir.7b03857 . hal-01696874

\section{HAL Id: hal-01696874 https://hal.science/hal-01696874}

Submitted on 31 Jan 2018

HAL is a multi-disciplinary open access archive for the deposit and dissemination of scientific research documents, whether they are published or not. The documents may come from teaching and research institutions in France or abroad, or from public or private research centers.
L'archive ouverte pluridisciplinaire HAL, est destinée au dépôt et à la diffusion de documents scientifiques de niveau recherche, publiés ou non, émanant des établissements d'enseignement et de recherche français ou étrangers, des laboratoires publics ou privés.

\section{다(1)(2)}

Distributed under a Creative Commons Attribution - ShareAlikel 4.0 International 


\title{
Viscocapillary Response of Gas Bubbles Probed by Thermal Noise Atomic Force Measurement
}

\author{
Yuliang Wang, ${ }^{\dagger}$ Binglin Zeng, ${ }^{\dagger}{ }^{\dagger}$ Hadush Tedros Alem, ${ }^{\dagger}$ Zaicheng Zhang, ${ }^{\ddagger}$ Elisabeth Charlaix, ${ }^{\S}$ \\ and Abdelhamid Maali*,*0 \\ ${ }^{\dagger}$ School of Mechanical Engineering and Automation, Beihang University, 37 Xueyuan Rd., Haidian District, Beijing 100191, China \\ ${ }^{\ddagger}$ Université de Bordeaux \& CNRS, LOMA, UMR 5798, F 33400 Talence, France \\ ${ }^{\S}$ LIPHY, Université Grenoble Alpes, F 38000 Grenoble, France
}
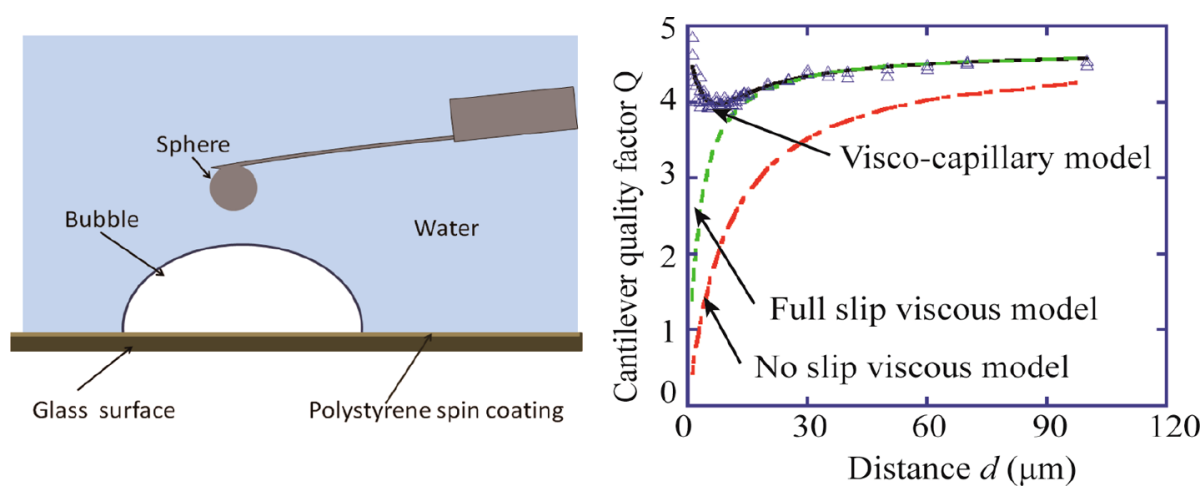

\begin{abstract}
We present thermal noise measurements of a vibrating sphere close to microsized air bubbles in water with an atomic force microscope. The sphere was glued at the end of a cantilever with a resonance frequency of few $\mathrm{kHz}$. The subangstrom thermal motion of the microsphere reveals an elastohydrodynamic coupling between the sphere and the air bubble.

The results are in perfect agreement with a model incorporating macroscopic capillarity and fluid flow on the bubble surface with full slip boundary conditions.
\end{abstract}

\section{INTRODUCTION}

Recently, much progress has been achieved for fluid flow in the vicinity of solid-liquid interfaces. ${ }^{1-3}$ Colloidal probe atomic force microscopy (AFM) is one of the techniques used to characterize fluid flows on the nanoscale. ${ }^{3}$

Liquid-gas interfaces introduced by microsized air bubbles in water are gaining increasing attention in the study of flow dynamics, especially on the micro/nanoscale. Assuming the shear stress continues at liquid-gas interfaces, the much lower viscosity of the gas theoretically makes them behave as shear free interfaces. As a result, liquid gas interfaces are thought to be good candidates for perfect slipping interfaces. ${ }^{4-7}$ However, Manor et al. have shown experimentally that the slip length at liquid-gas interfaces is moderate (a few of tens of nanometers) but not infinite, as expected. ${ }^{8-10}$ They measured the hydro dynamic force between two bubbles approaching each other at a constant velocity. In their analysis, they invoke the presence of impurity that contributes to the dynamic of fluid transport at the interfaces.

Maali et al. used a dynamic AFM to measure the hydrodynamic forces near air-water interfaces. ${ }^{11}$ In their experiment they measured the amplitude and phase of a vibrating cantilever approaching the liquid gas interface, from which the viscous and elastic hydrodynamic forces were derived. They showed that in a frequency range of a few tens of Hertz, the elastic force is due to the restoring effect of the Marangoni flow induced by transported surface impurities. As a consequence, the viscous hydrodynamic force shows a crossover from nonslip boundary conditions at low frequency to full slip boundary conditions at high frequency. Thanks to these low frequency dynamic measurements, they succeeded in evaluating the concentrations of surfaces impurities.

Although the increasing interest has been put on liquid-gas interfaces to study boundary $\operatorname{slip}^{7-14}$ or contact line dynamics, ${ }^{15}$ less is known about the dynamic response of bubbles under external excitation. We report flow measure ments at liquid gas interfaces using thermal noise AFM. The resonance frequency of the cantilever, in the range of a few kilohertz, exceeds by one order of magnitude the frequency range for which the liquid-gas surface elasticity is driven by the surfactant effect of surface impurities. In our experiments the flow near the interface is described by the full slip boundary conditions coupled to the elastic contribution of the capillary response of the bubble.

A Maxwell viscoelastic model with a spring and dashpot in series was used for modeling the hydrodynamic interaction 
between the sphere and the microsized bubbles. The experimental results fit well with the proposed model.

\section{MODELING OF THE HYDRODYNAMIC INTERACTION}

Hydrodynamic Damping. The fluid flow is normally described by continuity and Navies-Stokes equations. For low Reynolds number fluid flow under lubrication approximation, it can be written as

$$
\begin{aligned}
& \frac{\partial v_{z}}{\partial z}=-\frac{1}{r} \frac{\partial}{\partial r}\left(r v_{r}\right) \\
& \eta \frac{\partial^{2} v_{r}}{\partial z^{2}}=\frac{\partial p}{\partial r}
\end{aligned}
$$

where $p$ is the liquid pressure and $v_{z}$ and $v_{r}$ are vertical and radial velocities of fluid flow at radial and vertical location of $r$ and $z$ in a cylindrical coordinate system. A schematic diagram of hydrodynamic interaction measurement between a sphere and a microsized bubble is shown in Figure 1. On the sphere surface

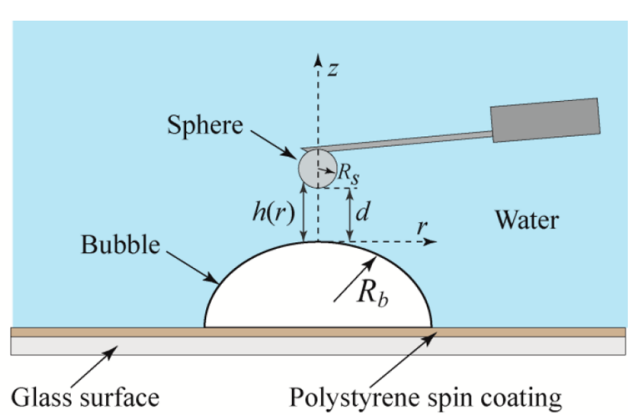

Figure 1. Schematic diagram of the hydrodynamic interaction measurement between a sphere and microsized bubble. The liquidgas interface was prepared by deposing a spherical bubble on polystyrene surface. A glass sphere glued at the end of the AFM cantilever is positioned at distance $d$ from the bubble.

the radial velocity of the fluid must satisfy the nonslip boundary conditions: $v_{r}(z=h)=0$ and vertical velocity is equal to the velocity of the vibrating sphere: $v_{z}(z=h)=\frac{\mathrm{d} Z}{\mathrm{~d} t}=i \omega Z$, where $Z$ is the instantaneous vertical position of the sphere. In Figure $1, h(r)=d+r^{2} / 2 R_{\text {eff }}$ is called confinement thickness, where $R_{\text {eff }}$ is the effective radius and is related to the radius of the sphere $R_{\mathrm{s}}$ and the bubble $R_{\mathrm{b}}$ as $R_{\text {eff }}=R_{\mathrm{s}} R_{b} /\left(R_{\mathrm{s}}+R_{\mathrm{b}}\right) .{ }^{16}$ On the full slip surface, we have the boundary conditions for the vertical and radial velocities: $\eta \frac{v_{r}(z=0)}{\partial z}=0$ and $v_{z}(z=0)=0$.

The liquid-gas interface was prepared by depositing a spherical bubble on polystyrene surface. A glass sphere glued at the end of the AFM cantilever is positioned at distance $d$ from the bubble.

By integrating eq 1 with respect to $z$ and using the boundary condition, we get

$$
v_{r}(z)=\frac{1}{\eta}\left[\frac{1}{2}\left(z^{2}-h^{2}\right) \frac{\partial p}{\partial r}\right]
$$

By integrating eq 2 with respect to $z$, we get the lubrication equation

$$
i \omega Z=\frac{1}{3 \eta r} \frac{\partial}{\partial r}\left(r h^{3} \frac{\partial p}{\partial r}\right)
$$

By multiplying both sides of eq 4 by $r$ and integrating twice with respect to $r$, we get the expression of the hydrodynamic pressure

$$
p(r)=-\frac{3 i \omega Z \eta R_{\mathrm{eff}}}{4\left(d+\frac{r^{2}}{2 R_{\mathrm{eff}}}\right)^{2}}
$$

The hydrodynamic interaction force is given by the integral of the hydrodynamic pressure over the substrate surface

$$
F_{\mathrm{h}}=2 \pi \int_{0}^{\infty} r p(r) \mathrm{d} r=-\frac{3 \pi i \omega Z \eta R_{\mathrm{eff}}^{2}}{2 d}
$$

By comparing eq 6 to the hydrodynamic force defined by $F_{\mathrm{h}}=-\gamma_{\mathrm{H}} \frac{\mathrm{d} Z}{\mathrm{~d} t}=-i \gamma_{\mathrm{H}} \omega Z$, we obtain the expression of the hydrodynamic damping for a full slip surface

$$
\omega \gamma_{\mathrm{H}}=\frac{3 \pi \omega \eta R_{\mathrm{eff}}^{2}}{2 d}
$$

In a similar way, we can calculate the hydrodynamic force in the case of the nonslip boundary conditions, $v_{r}(z=0)=0$ : $F_{\mathrm{h}}^{\mathrm{NS}}=-\frac{6 \pi i \omega Z \eta R_{\mathrm{eff}}^{2}}{d}$. The damping under the nonslip boundary condition is four times that for the full slip one. ${ }^{16}$

Bubble Stiffness. The capillary deformation $\xi$ of a bubble is related to hydrodynamic pressure $p(r)$ by the LaplaceYoung equation

$$
p(r)=\sigma \Delta \xi(r)=\frac{\sigma}{r} \frac{\partial}{\partial r}\left(r \frac{\partial \xi}{\partial r}\right)
$$

where the hydrodynamic pressure $p(r)$ is given by eq 5 and $\sigma$ is the water surface tension. By substituting eq 5 into eq 8 and integrating eq 8 , we obtain

$$
\frac{\mathrm{d} \xi}{\mathrm{d} r}=\frac{-3 i \omega Z \eta R_{\mathrm{eff}} r}{8 \sigma d\left(d+\frac{r^{2}}{2 R_{\mathrm{eff}}}\right)}
$$

Integrating again between $r=0$ and $r=R_{\mathrm{b}}$, where $R_{\mathrm{b}}$ is the bubble radius, and assuming $\xi\left(r=R_{\mathrm{b}}\right)=0$, we get

$$
\begin{gathered}
\xi(r=0)=\xi_{0}=\frac{3 i \omega Z \eta R_{\mathrm{eff}}^{2}}{2 d \sigma} \ln \left(1+\frac{R_{\mathrm{b}}^{2}}{2 R_{\mathrm{eff}} d}\right) \approx \frac{3 i \omega Z \eta R_{\mathrm{eff}}^{2}}{2 d \sigma} \\
\ln \left(\frac{R_{\mathrm{b}}^{2}}{2 R_{\mathrm{eff}} d}\right)
\end{gathered}
$$

The bubble stiffness $k_{\mathrm{b}}$ is defined as $F_{\mathrm{h}}=k_{\mathrm{b}} \xi_{0}$, and from eqs 6 and 10 , we get

$$
k_{\mathrm{b}}=\frac{F_{\mathrm{h}}}{\xi_{0}}=\frac{4 \pi \sigma}{\ln \left(\frac{R_{\mathrm{b}}^{2}}{2 R_{\mathrm{eff}} D}\right)}
$$

Note here that in the derivation of the bubble stiffness we have assumed a flat bubble. This assumption is thought to be valid because in our case the radius of the bubble is much larger than the radius of the colloidal sphere.

Viscocapillary Interaction. The viscoelastic response of bubbles can be modeled using the Maxwell model: spring and 
dashpot (damping) in series (see Figure 2). In this simple model, the viscoelastic modulus is given by

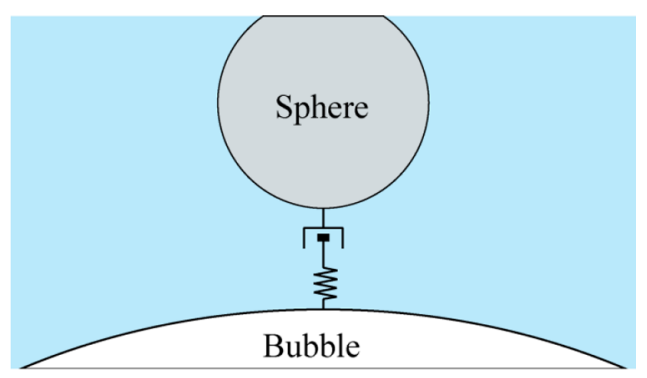

Figure 2. Equivalent model of the viscoelastic response of the microsized bubble: spring and dashpot in series.

$$
G^{\prime}+j G^{\prime \prime}=\frac{1}{\frac{1}{k_{\mathrm{b}}}+\frac{1}{i \omega \gamma_{\mathrm{H}}}}=\frac{k_{\mathrm{b}}\left(\omega \gamma_{\mathrm{H}}\right)^{2}}{k_{\mathrm{b}}^{2}+\left(\omega \gamma_{\mathrm{H}}\right)^{2}}+i \frac{k_{\mathrm{b}}^{2} \omega \gamma_{\mathrm{H}}}{k_{\mathrm{b}}^{2}+\left(\omega \gamma_{\mathrm{H}}\right)^{2}}
$$

where $\gamma_{\mathrm{H}}$ is the damping coefficient of hydrodynamic interaction calculated for a perfect slip solid surface and $k_{\mathrm{b}}$ is bubble stiffness.

By substituting eqs 7 and 11 into eq 12, the modulus of the elastic component $G^{\prime}$ and the dissipative component $G^{\prime \prime}$ of the interaction can be given as

$$
\begin{aligned}
G^{\prime} & =\frac{3 \pi \eta \omega R_{\mathrm{eff}}^{2}}{2 d} \frac{\frac{3 \eta \omega R_{\mathrm{eff}}^{2}}{8 \sigma d} \ln \left(\frac{R_{\mathrm{b}}^{2}}{2 R_{\mathrm{eff}} d}\right)}{1+\left[\frac{3 \eta \omega R_{\mathrm{eff}}^{2}}{8 \sigma d} \ln \left(\frac{R_{b}^{2}}{2 R_{\mathrm{eff}} d}\right)\right]^{2}} \\
G^{\prime \prime} & =\frac{3 \pi \eta \omega R_{\mathrm{eff}}^{2}}{2 d} \frac{1}{1+\left[\frac{3 \eta \omega R_{\mathrm{eff}}^{2}}{8 \sigma d} \ln \left(\frac{R_{\mathrm{b}}^{2}}{2 R_{\mathrm{eff}} d}\right)\right]^{2}}
\end{aligned}
$$

From eqs $13 \mathrm{a}$ and $13 \mathrm{~b}$, one can expect that there should be two asymptotic behaviors

(1) Far from the bubble surface: $d \gg \frac{3 \eta \omega R_{\mathrm{eff}}^{2}}{8 \sigma}, G^{\prime} \gg G^{\prime \prime}$ (viscous regime) with

$$
G^{\prime \prime} \approx \frac{3 \pi \eta \omega R_{\mathrm{eff}}^{2}}{2 d}
$$

and

$$
G^{\prime} \approx \frac{9 \pi\left(\eta \omega R_{\mathrm{eff}}^{2}\right)^{2}}{16 \sigma d^{2}} \ln \left(\frac{R_{\mathrm{b}}^{2}}{2 R_{\mathrm{eff}} d}\right)
$$

(2) Close to the bubble surface: $d \ll \frac{3 \eta \omega R_{\text {eff }}^{2}}{8 \sigma}, G^{\prime} \ll G^{\prime \prime}$ (elastic regime) with

$$
G^{\prime \prime} \approx \frac{32 \pi \sigma^{2} d}{3 \eta \omega R_{\mathrm{eff}}^{2}\left[\ln \left(\frac{R_{\mathrm{b}}^{2}}{2 R_{\mathrm{eff}} d}\right)\right]^{2}}
$$

and

$$
G^{\prime} \approx \frac{4 \pi \sigma}{\ln \left(\frac{R_{\mathrm{b}}^{2}}{2 R_{\mathrm{eff}} d}\right)}
$$

Quality Factor and Resonance Frequency of the Cantilever. For a vibrating colloidal cantilever close to liquid-gas interfaces of bubbles, the motion equation $z(t)$ of the tip can be described using the oscillator model

$$
m^{*} \ddot{z}+\gamma_{\text {bulk }} \dot{z}+k_{c} z=F_{\text {Noise }}+F_{\mathrm{h}}
$$

where $m^{*}$ is the effective mass of the cantilever, $k_{c}$ is the cantilever force constant, $\gamma_{\text {bulk }}$ is the bulk damping coefficient far from the surface, $F_{\text {Noise }}$ is the random thermal noise force, and $F_{\mathrm{h}}$ is the hydrodynamic interaction force between the sphere and the bubble

$$
F_{\mathrm{h}}=-\left(G^{\prime}+j G^{\prime \prime}\right) z
$$

The equation of the motion becomes

$$
m^{*} \ddot{z}+\gamma_{\text {bulk }} \dot{z}+k_{c} z+G^{\prime} z+i G^{\prime \prime} z=F_{\text {Noise }}
$$

or

$$
\left[-m^{*} \omega^{2}+k_{\mathrm{c}}+G^{\prime}+i \omega\left(\gamma_{\text {bulk }}+\frac{G^{\prime \prime}}{\omega}\right)\right] z=F_{\text {Noise }}
$$

Thus the spectral density of the thermal noise can be given by ${ }^{17}$

$$
S D(\omega)=\frac{c_{1}}{\left[\omega_{\mathrm{r}}(d)^{2}-\omega^{2}\right]^{2}+\left[\frac{\omega_{\mathrm{r}}^{\text {bulk }} \omega}{Q(d)}\right]^{2}}+c_{2}
$$

where $c_{1}$ and $c_{2}$ are two fitting constants and $\omega_{\mathrm{r}}(d)$ and $Q(d)$ are the resonance frequency and the quality factor at a distance $d$ from the bubble, respectively. They are related to the bulk values by

$$
\begin{gathered}
Q(d) \approx \frac{Q_{\text {bulk }}}{1+\frac{3 \pi \eta R_{\text {eff }}^{2}}{2 \gamma_{\text {bulk }}^{d}} \frac{1}{1+\left[\frac{3 \eta \omega_{\mathrm{r}}^{\text {bulk }}}{8 \sigma d} \text { eff }^{2} \ln \left(\frac{R_{\mathrm{b}}^{2}}{2 R_{\text {eff }}}\right)\right]^{2}}} \\
\omega_{\mathrm{r}}(d) \approx \omega_{\mathrm{r}}^{\text {bulk }}+\frac{\pi \eta \omega_{\mathrm{r}}^{\text {bulk }}\left(3 \eta \omega_{\mathrm{r}}^{\text {bulk }} R_{\text {eff }}^{2}\right)^{2}}{32 k_{\mathrm{c}} \sigma d^{2}} \\
\frac{\ln \left(\frac{R_{\mathrm{b}}^{2}}{2 R_{\text {eff }} d}\right)}{1+\left[\frac{3 \eta \omega_{\mathrm{r}}^{\text {bulk }} R_{\text {eff }}^{2}}{8 \sigma \sigma d} \ln \left(\frac{R_{\mathrm{b}}^{2}}{2 R_{\text {eff }} d}\right)\right]^{2}}
\end{gathered}
$$

where the bulk values are given by $\omega_{r}^{\text {bulk }}=\sqrt{\frac{k_{c}}{m^{*}}}$ and $\gamma_{\text {bulk }}=\frac{m^{*} \omega_{\mathrm{r}}^{\text {bulk }}}{Q_{\text {bulk }}}=\frac{k_{\mathrm{c}}}{\omega_{\mathrm{r}}^{\text {bulk }} Q_{\text {bulk }}}$.

At enough large distance between the sphere and the bubble $\left(d \gg \frac{3 \eta \omega R_{\text {eff }}^{2}}{8 \sigma}\right)$ for which the capillary response can be neglected, the resonance frequency and the quality factor reduce to $Q(d) \approx Q_{\text {bulk }} /\left(1+\frac{3 \pi \eta R_{\text {eff }}^{2}}{2 \gamma_{\text {bulk }} d}\right)$ and $\omega_{\mathrm{r}}(d) \approx \omega_{\mathrm{r}}^{\text {bulk }}$.

\section{EXPERIMENTAL METHODS AND RESULTS}

In this study, the liquid-gas interface was prepared by placing a spherical air bubble with a radius $R_{\mathrm{b}}=220.0 \pm 4.0 \mu \mathrm{m}$ on PS surface (substrate) using a microsyringe. The bubble radius was measured with an optical microscope. The experiment was performed using an AFM (Resolve, Bruker, USA). A spherical borosilicate particle (MO Sci Corporation) with a radius $R_{\mathrm{s}}=44.0 \pm 0.8 \mu \mathrm{m}$ was used. The sphere was glued to the end of a silicon cantilever (NP, Bruker) using epoxy (Araldite, Bostik, Coubert). The effective radius for hydro 
dynamic interaction on the bubble equals $R_{\text {eff }}=R_{\mathrm{s}} R_{\mathrm{b}} /\left(R_{\mathrm{s}}+R_{\mathrm{b}}\right)=36.7$ $\pm 0.7 \mu \mathrm{m}$. After the sphere was glued to the end of the cantilever, the cantilever stiffness was calibrated using the thermal noise method. The obtained stiffness was $k_{\mathrm{c}}=0.35 \pm 0.02 \mathrm{~N} / \mathrm{m}$. The bulk resonance frequency, quality factor, and damping coefficient are found to be $\omega_{\mathrm{r}}^{\text {bulk }} / 2 \pi=3.48 \pm 0.05 \mathrm{kHz}, Q_{\text {bulk }}=4.7 \pm 0.1$, and $\gamma_{\text {bulk }}=k_{\mathrm{c}} /$ $\left(\omega_{\mathrm{r}}^{\text {bulk }} Q_{\text {bulk }}\right)=(3.4 \pm 0.3) \times 10^{-6} \mathrm{~N} \mathrm{~m}^{-1} \mathrm{~s}$.

The AFM cantilever remained stationary during measurement in this study. It was only driven by thermal noise. The maximum thermal oscillation amplitude was $<1.0 \mathrm{~nm}$. Therefore, the influence of cantilever oscillation on the separation distance can be neglected.

The distance between the sphere and the bubble was controlled by the integrated stage step motor. Each separation distance was adjusted by displacing the cantilever vertically using the step motor with reproducibility $<0.1 \mu \mathrm{m}$. The position at which the cantilever deflection signal changed was taken as contact point, namely, the zero separation distance.

The thermal noise signal of the cantilever deflection was acquired using an analog to digital (A/D) acquisition board (PCI 4462, National Instrument, USA) with a sampling rate of $80 \mathrm{kHz}$. With the data, the spectral density of the thermal noise was calculated. An example of the obtained thermal noise signal was shown in Figure 3.

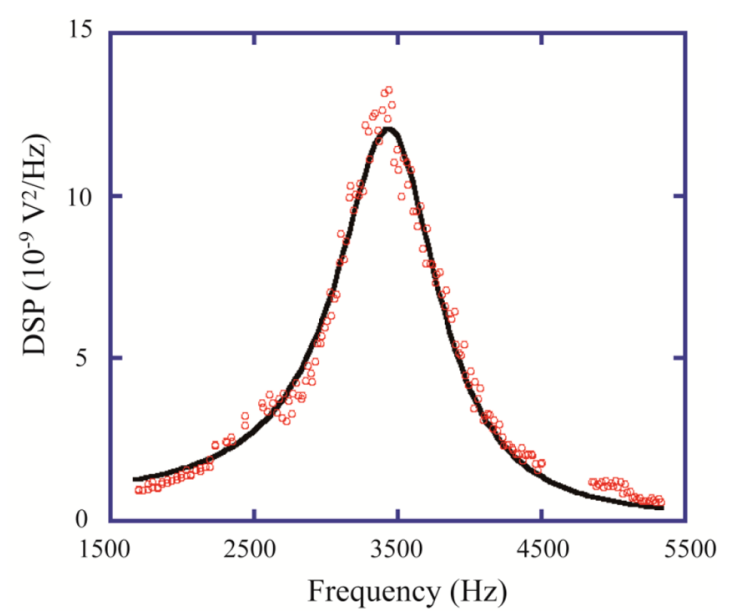

Figure 3. Example of the thermal noise density spectra obtained for the sphere at distance $14 \mu \mathrm{m}$ from the bubble surface. The solid line is the fitting curve by eq 18 .
At each distance between the bubble and the sphere, the spectral density of the thermal noise was fitted using eq 18, through which the quality factor and the resonance frequency can be obtained.

Besides liquid-air interfaces, here a mica surface was used to provide hydrophilic solid-liquid interface for comparison. ${ }^{18-20}$ The normalized quality factor (with respect to bulk values) versus the distance for the colloidal probe on mica is shown in Figure 4A. The damping on mica surface is given by $6 \pi \eta R_{s}^{2} / d$ using a nonslip boundary condition. ${ }^{18-20}$ The quality factor on mica can then be fitted using the expression: $Q^{\mathrm{NS}}(d)=Q_{\text {bulk }} /\left(1+\frac{6 \pi \eta R_{\mathrm{s}}{ }^{2}}{\gamma_{\text {bulk }}{ }^{d}}\right)$ (see Figure 4A).

The quality factor measured on the bubble is presented in Figure 4B, which is different from the results on the mica surface. The quality factor starts from a bulk value of 4.7 and decreases with the decreasing separation distance between the bubble and the sphere. After the quality factor reaches a minimum value, it begins to increase with decreasing separation distance. At large separation the data coincide with the theoretical curve calculated assuming full slip boundary conditions on the bubble surface. This result is in agreement with the study of Maali et al. ${ }^{11}$ Indeed the cantilever resonance frequency is very large compared with the frequency that characterizes the contribution of impurities to the flow $\nu_{\mathrm{c}}=\Pi_{0} / 16 \pi \eta R_{\mathrm{eff}}$, where $\Pi_{0}$ is the impurity surface pressure. (For a similar preparation protocol, bubble deposited on polystyrene surface in pure water, the impurity surface pressure value was estimated by Maali et al. ${ }^{11}$ to be $\Pi_{0} \approx 0.25$ $\mathrm{mN} / \mathrm{m}$, and thus $\left.v_{\mathrm{c}} \approx 135 \mathrm{~Hz} \ll \omega_{\mathrm{r}}^{\text {bulk }} / 2 \pi\right)$.

At small separation distance, the bubble elastic deformation accommodates the motion of the sphere. ${ }^{21-24}$ This cancels the viscous flow of the liquid, leading to the increased quality factor.

Moreover the viscocapillary hydrodynamic coupling induces a shift of the resonance frequency of the cantilever, as expected by eq 20 . The change of resonance frequency of the colloidal probe with changing separation distance is shown in Figure 5. The resonance frequency increases sharply as the sphere is very close to the bubble surface.

\section{CONCLUSIONS}

The viscocapillary response of microsized air bubbles to thermally driven vibrating spheres was investigated in this study. By measuring thermal noise signal of the colloidal probe attached to the AFM cantilever, the resonance frequency and quality factor of the vibrating cantilever were obtained at different separation distance between the sphere and the bubble. The subangstrom thermal motion of the microsphere
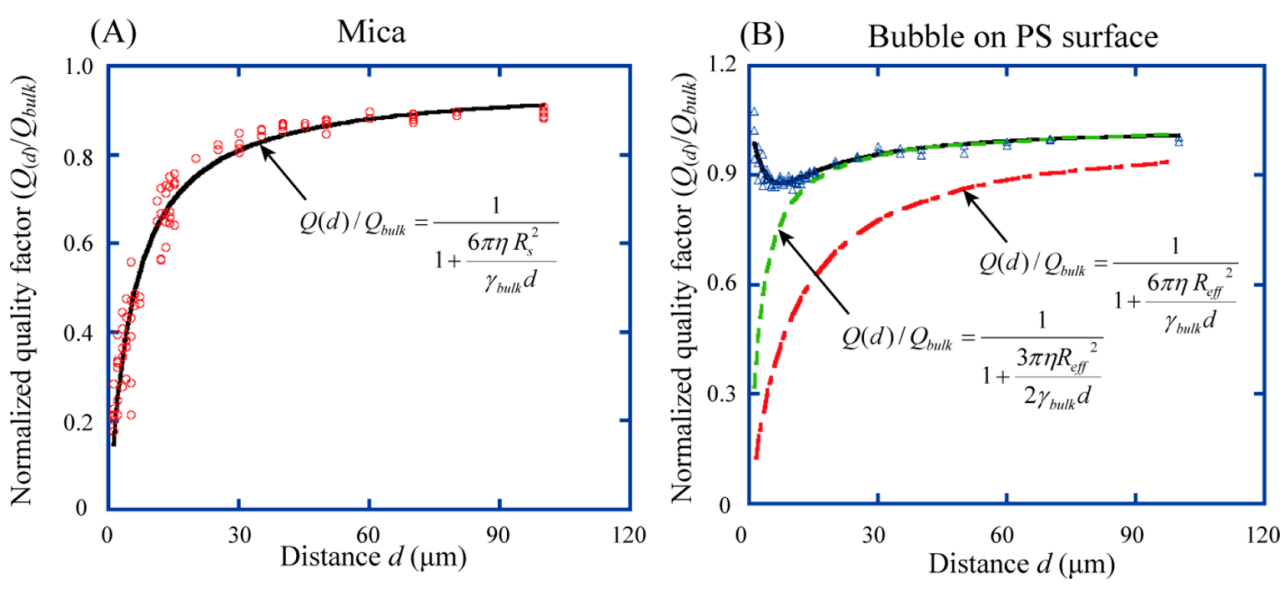

Figure 4. Normalized quality factor versus the distance fitted for different density spectra. (A) Result on mica surface. The data were fitted with $Q(d) / Q_{\text {bulk }}=\left(1+\frac{6 \pi \eta R_{\mathrm{s}}{ }^{2}}{\gamma_{\text {bulk }}{ }^{d}}\right)^{-1}$ of the nonslip boundary condition (solid curve). (B) Results on the bubble surface. The dark solid lines are the fitting curves using eq 19, and the green dotted line and the red dashed dotted line are the theoretical simulating curves for quality factor of the full $\operatorname{slip} Q(d) / Q_{\text {bulk }}=\left(1+\frac{3 \pi \eta R_{\text {eff }}{ }^{2}}{2 \gamma_{\text {bulk }^{d}}}\right)^{-1}$ and nonslip $Q(d) / Q_{\text {bulk }}=\left(1+\frac{6 \pi \eta R_{\text {eff }}{ }^{2}}{\gamma_{\text {bulk }} d}\right)^{-1}$, respectively, neglecting capillary response. 


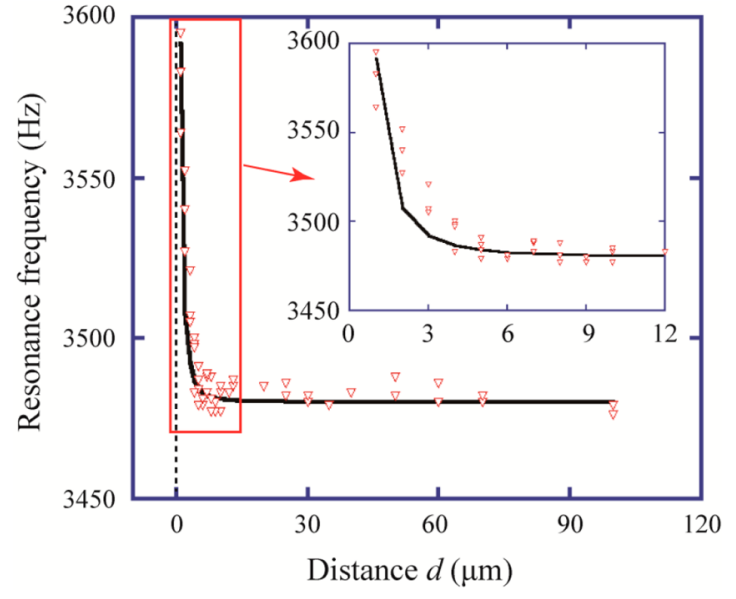

Figure 5. Resonance frequency of the cantilever versus the distance between the sphere and the bubble. The data were fitted with eq 20 . The inset figure is the zoomed one for the area selected by the rectangular box.

reveals an elastohydrodynamic coupling between the sphere and the air bubble. A mathematical model with a spring and dashpot in series was developed. This model combines the macroscopic capillarity and fluid flow on the bubble surface with full slip boundary conditions. The experimental results fit well with the theoretical predictions of the developed model.

\section{AUTHOR INFORMATION}

\section{Corresponding Author}

*E mail: abdelhamid.maali@u bordeaux.fr.

\section{ORCID}

Yuliang Wang: 0000000161304321

Binglin Zeng: 0000000187291944

Abdelhamid Maali: 0000000294596081

\section{Notes}

The authors declare no competing financial interest.

\section{ACKNOWLEDGMENTS}

We thank R. Boisgard, A. Wurger, T. Salez, and Y. Amarouchene for fruitful discussions. Y.W. acknowledges the financial support by National Natural Science Foundation of China (grant no. 51775028) and Beijing Natural Science Foundation (grant no. L150002). Z.Z. acknowledges financial support from the Chinese Scholarship Council.

\section{REFERENCES}

(1) Bocquet, L.; Charlaix, E. Nanofluidics, from bulk to interfaces. Chem. Soc. Rev. 2010, 39 (3), 1073-1095.

(2) Lauga, E.; Brenner, M.; Stone, H., Microfluidics: The No Slip Boundary Condition. In Springer Handbook of Experimental Fluid Mechanics; Springer: 2007; pp 1219-1240.

(3) Neto, C.; Evans, D. R.; Bonaccurso, E.; Butt, H. J.; Craig, V. S. Boundary slip in Newtonian liquids: a review of experimental studies. Rep. Prog. Phys. 2005, 68 (12), 2859.

(4) de Gennes, P. G. On fluid/wall slippage. Langmuir 2002, 18 (9), 3413-3414.

(5) Zhu, Y.; Granick, S. Rate dependent slip of Newtonian liquid at smooth surfaces. Phys. Rev. Lett. 2001, 87 (9), 096105.

(6) Ybert, C.; Barentin, C.; Cottin Bizonne, C.; Joseph, P.; Bocquet, L. Achieving large slip with superhydrophobic surfaces: Scaling laws for generic geometries. Phys. Fluids 2007, 19 (12), 123601.
(7) Tretheway, D. C.; Meinhart, C. D. A generating mechanism for apparent fluid slip in hydrophobic microchannels. Phys. Fluids 2004, 16 (5), 1509-1515.

(8) Manor, O.; Vakarelski, I. U.; Tang, X.; O’Shea, S. J.; Stevens, G. W.; Grieser, F.; Dagastine, R. R.; Chan, D. Y. Hydrodynamic boundary conditions and dynamic forces between bubbles and surfaces. Phys. Rev. Lett. 2008, 101 (2), 024501.

(9) Manor, O.; Vakarelski, I. U.; Stevens, G. W.; Grieser, F.; Dagastine, R. R.; Chan, D. Y. Dynamic forces between bubbles and surfaces and hydrodynamic boundary conditions. Langmuir 2008, 24 (20), 11533-11543.

(10) Manor, O.; Chan, D. Y. Influence of surfactants on the force between two bubbles. Langmuir 2010, 26 (2), 655-662.

(11) Maali, A.; Boisgard, R.; Chraibi, H.; Zhang, Z.; Kellay, H.; Würger, A. Viscoelastic Drag Forces and Crossover from No Slip to Slip Boundary Conditions for Flow near Air Water Interfaces. Phys. Rev. Lett. 2017, 118 (8), 084501.

(12) Hyväluoma, J.; Harting, J. Slip flow over structured surfaces with entrapped microbubbles. Phys. Rev. Lett. 2008, 100 (24), 246001.

(13) Schäffel, D.; Koynov, K.; Vollmer, D.; Butt, H. J.; Schönecker, C. Local flow field and slip length of superhydrophobic surfaces. Phys. Rev. Lett. 2016, 116 (13), 134501.

(14) Bolognesi, G.; Cottin Bizonne, C.; Pirat, C. Evidence of slippage breakdown for a superhydrophobic microchannel. Phys. Fluids 2014, 26 (8), 082004

(15) Wang, Y.; Wang, H.; Bi, S.; Guo, B. Nano Wilhelmy investigation of dynamic wetting properties of AFM tips through tip nanobubble interaction. Sci. Rep. 2016, 6, 30021.

(16) Vinogradova, O. I. Drainage of a thin liquid film confined between hydrophobic surfaces. Langmuir 1995, 11 (6), 2213-2220.

(17) Bowles, A. P.; Honig, C. D.; Ducker, W. A. No Slip Boundary Condition for Weak Solid- Liquid Interactions. J. Phys. Chem. C 2011, 115 (17), 8613-8621.

(18) Honig, C. D.; Ducker, W. A. No slip hydrodynamic boundary condition for hydrophilic particles. Phys. Rev. Lett. 2007, 98 (2), 028305 .

(19) Honig, C. D.; Ducker, W. A. Thin film lubrication for large colloidal particles: experimental test of the no slip boundary condition. J. Phys. Chem. C 2007, 111 (44), 16300-16312.

(20) Maali, A.; Wang, Y.; Bhushan, B. Evidence of the no slip boundary condition of water flow between hydrophilic surfaces using atomic force microscopy. Langmuir 2009, 25 (20), 12002-12005.

(21) Steinberger, A.; Cottin Bizonne, C.; Kleimann, P.; Charlaix, E. Nanoscale flow on a bubble mattress: Effect of surface elasticity. Phys. Rev. Lett. 2008, 100 (13), 134501.

(22) Leroy, S.; Charlaix, E. Hydrodynamic interactions for the measurement of thin film elastic properties. J. Fluid Mech. 2011, 674, 389-407.

(23) Leroy, S.; Steinberger, A.; Cottin Bizonne, C.; Restagno, F.; Léger, L.; Charlaix, É. Hydrodynamic interaction between a spherical particle and an elastic surface: a gentle probe for soft thin films. Phys. Rev. Lett. 2012, 108 (26), 264501.

(24) Villey, R.; Martinot, E.; Cottin Bizonne, C.; Phaner Goutorbe, M.; Léger, L.; Restagno, F.; Charlaix, E. Effect of surface elasticity on the rheology of nanometric liquids. Phys. Rev. Lett. 2013, 111 (21), 215701. 